\title{
FIFTEEN YEARS OF SINGLE CENTER EXPERIENCE WITH STEM CELL TRANSPLANTATION FOR MULTIPLE MYELOMA: A RETROSPECTIVE ANALYSIS
}

\author{
Jakub Radocha, Vladimír Maisnar, Alžběta Zavřelová, Melanie Cermanová, Miriam Lánská, Miloslav Kmoníček, \\ Ladislav Jebavý, Milan Bláha, Jaroslav Malý, Pavel Žák
}

Charles University in Prague, Faculty of Medicine in Hradec Králové, and University Hospital in Hradec Králové, Czech Republic: 4th Department of Hematology

\begin{abstract}
Summary: Introduction: Autologous stem cell transplantation (ASCT) became standard of care for patients with multiple myeloma (MM) under the age of 65 years. We routinely perform ASCT for newly diagnosed MM since 1996 in our department. Patients and methods: We retrospectively analyzed all 285 transplants in 185 patients done for MM from January 1996 till December 2010. We analyzed overall survival (OS) and progression-free survival (PFS) regarding conditioning, stage, complete or very good partial remission (CR, VGPR) achievement, renal impairment, single vs. double transplant. Results: Estimated 10-years survival of the whole set of patients is 39\% (median survival 95 months). Patients with renal impairment show same OS as those without $(\mathrm{p}=0.22)$. Patients show similar overall survival and event free survival regardless of type of transplant. We observed better outcome in terms of overall survival in patients treated with new drugs $(p=0.0014)$. Reaching CR or VGPR was not translated into better OS $(p=0.30)$ and EFS $(\mathrm{p}=0.10)$. Also stage of the disease and whether single or double transplant was used did not make any significant difference in the outcome. Conclusion: Stem cell transplantation greatly improved outcome of patients with MM. Poor outcome of allogeneic transplantation in our group of patients is related to high transplant related mortality $(20 \%$ vs. $0 \%$ ) and unexpected high relapse rate. There is a trend towards better survival, when new drugs are incorporated at any time in the course of the disease. This fact supports hypothesis that use of these drugs with ASCT should translate into better long-term outcome.
\end{abstract}

Key words: Multiple myeloma; Transplantation; Therapy; New drugs; Results

\section{Introduction}

Multiple myeloma is the second most frequent hematologic malignancy responsible for about 86,000 new cases worldwide each year (1). The symptomatic disease is characterised by the presence of increased number of clonal plasma cells in the bone marrow (more than 10\%), presence of monoclonal immunoglobulin and end-organ damage (renal impairment, anemia, hyperkalcemia, bone disease, known as CRAB) (2). Although benefit from modern treatment is large, multiple myeloma is still considered to be incurable disease. However, in recent decade, a great improvement in overall survival has been reached. In early $60 \mathrm{~s}$, Melphalan was introduced as one of the first drugs that shown promising efficacy against myeloma cells (3). Since then until the middle 90 s no treatment was truly able to prolong life of myeloma patients and reached satisfactory response rate. One of the milestones was introduction of high-dose therapy followed by autologous stem cell transplantation. It has been shown in several studies, that autologous stem cell transplantation is able to not only prolong progression free survival of the patients, but also possibly prolong overall survival of the patients $(4,5)$. Since then a lot further research was done and several new drugs have been introduced to the treatment of myeloma patients. Especially thalidomide and bortezomib have shown significant activity against multiple myeloma but the impact of these drugs on overall survival remained controversial for couple of years. Now it seems clear that addition of new drugs to standard chemotherapy as well as autologous stem cell transplantation is of high benefit to all patients (6). Combining new treatments with high-dose therapy seems to be very promising in long term disease control and even possible cure within a small portion of patients.

\section{Patients and methods}

We retrospectively analyzed all consecutive multiple myeloma patients who undergone any type of transplantation at the 2nd Department of Medicine - Division of Clinical Hematology at University Hospital in Hradec Králové from January 1996 (beginning of the transplant programme in $\mathrm{MM}$ at our institution and in the Czech Republic) till December 2010. Diagnosis of multiple myeloma was based 
on IMWG criteria (2), staging of patients was done using Durie-Salmon criteria (7). Treatment responses were evaluated only in patients who had a follow-up longer than 6 weeks. Response to therapy, progression or relapse of disease were defined using the standard European Group for Blood and Marrow Transplantation (EBMT) criteria (8). Complete responses (CRs) were confirmed by negative serum immunofixation. Because of later introduction of more modern staging systems (such as ISS) these were not included in the analysis. In all patients the response rate, overall survival, progression free survival was calculated. Data were then analyzed by Kaplan-Meier survival analysis in different subgroups. We analyzed overall survival (OS) and progression-free survival (PFS) regarding conditioning, stage, complete remission achievement, renal impairment, single vs. double transplant and use of new drugs (thalidomide, bortezomib and lenalidomide). P value $<0.05$ was considered to be statistically significant. Microsoft Excel 2007 and MedCalc 9.5.2.0 were used for statistical analysis.

\section{Results}

We identified 185 patients who underwent 285 transplant procedures at our institution during the given time period. There were 99 males and 86 females. The median age was 57 years (28-75 years range). Detailed summary of the disease types and patients is summarized in table 1 . The vast majority of transplants were autologous procedures. 255 procedures were conditioned using Melphalan $200 \mathrm{mg} / \mathrm{m}^{2}, 15$ procedures using Melphalan $100 \mathrm{mg} / \mathrm{m}^{2}$ and 15 transplants were allogeneic in upfront auto-allo setting. Most frequent conditioning in this group was non-myeloablative so called "Slavin protocol" (Busulphan, Fludarabine $+/$ - Antithymocytic globuline) (9). The transplant types and conditioning regimens are summarized in table 2 .

Tab. 1: Patient characteristics

\begin{tabular}{|l|c|}
\hline \multicolumn{1}{|c|}{ Total No. of patients } & 185 \\
\hline & 86 females \\
\hline Age & 99 males \\
\hline average & $58(28-75)$ \\
\hline median & 57 \\
\hline Stage & \\
\hline IIA & 80 \\
\hline IIB & 5 \\
\hline IIIA & 76 \\
\hline
\end{tabular}

\begin{tabular}{|l|c|}
\hline IIIB & 20 \\
\hline Plasma cell leukemia & 4 \\
\hline M-protein type & 13 \\
\hline IgA lambda & 18 \\
\hline IgA kappa & 43 \\
\hline IgG lambda & 93 \\
\hline IgG kappa & 2 \\
\hline IgD lambda & 9 \\
\hline kappa free & 3 \\
\hline lambda free & 3 \\
\hline biclonal & 1 \\
\hline non-secretory & \\
\hline
\end{tabular}

Tab. 2: Transplant types and TRM in our data file.

\begin{tabular}{|l|c|}
\hline \multicolumn{1}{|c|}{ Total No. of transplants } & $\mathbf{2 8 5}$ \\
\hline Mel200 & 255 \\
\hline Mel100 & 15 \\
\hline Auto+RIC allo & 13 \\
\hline Syngenic allo & 1 \\
\hline Allo ablative & 1 \\
\hline Transplant related mortality & \\
\hline autologous & $0 / 255$ \\
\hline allogenic & $3 / 15$ \\
\hline
\end{tabular}

Estimated 10-years survival of the whole set of patients is $39 \%$ (median survival 95 months, figure 1). Regardless of type of transplantation, OS and EFS were the same for all the groups of patients $(p=0.39)$ (figure 2$)$. The same was found to be true for patients with renal insufficiency versus those who did not have it $(p=0.22)$. Here we need to point out that this is only rough division into patients with stage A versus stage B myeloma according to Durie-Salmon staging system. Thus these results do not reflect the possible reversibility of renal impairment after induction treatment. On the other hand, we transplant also patients undergoing dialysis at the time of transplant and these are also included in the analysis, which means that transplant is also viable option for these patients. Patients with stage II versus stage III disease $(p=0.92)$ or patients who reached $\mathrm{CR}$ or not $(\mathrm{p}=0.31)$ (figures $3-5)$. Significant difference in 


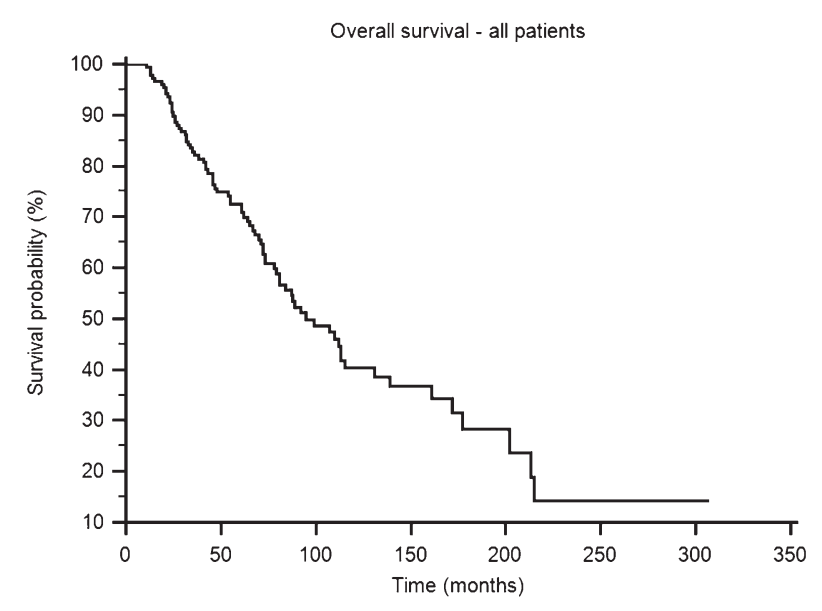

Fig. 1: Overall survival of all patients included in the analysis

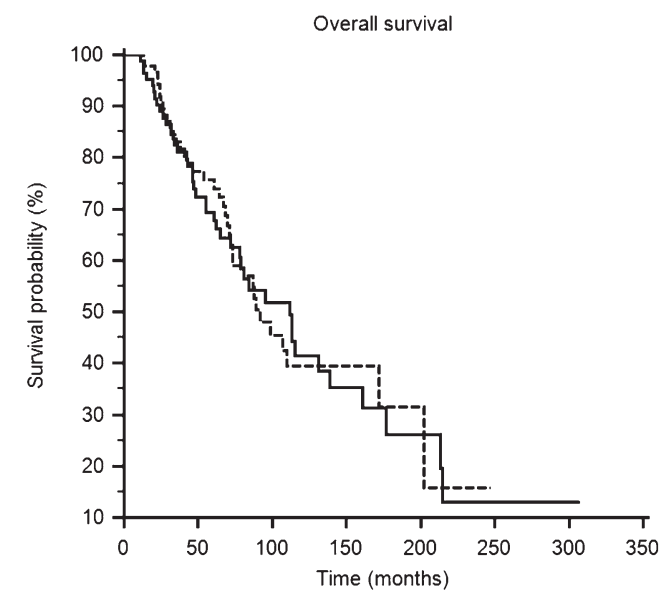

Fig. 3: Overall survival in patients with stage II versus stage III disease (Yes: patient with stage III disease, No: all other patients)

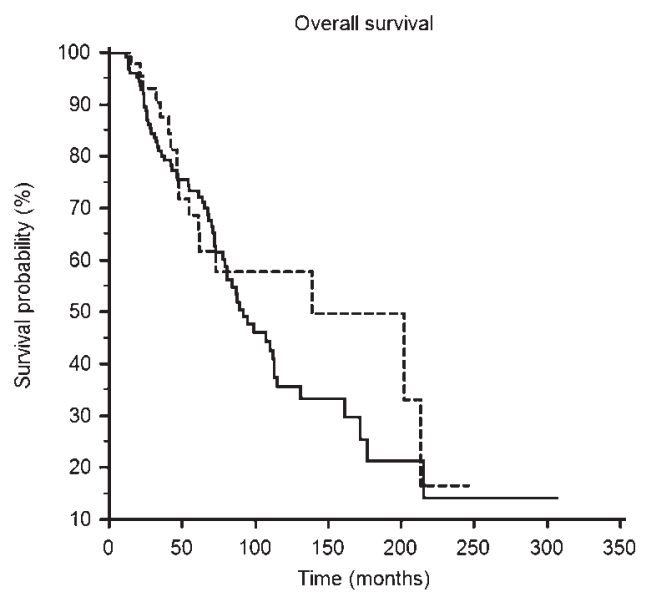

Fig. 5: Overall survival in patients with $\mathrm{CR}$ versus no $\mathrm{CR}$

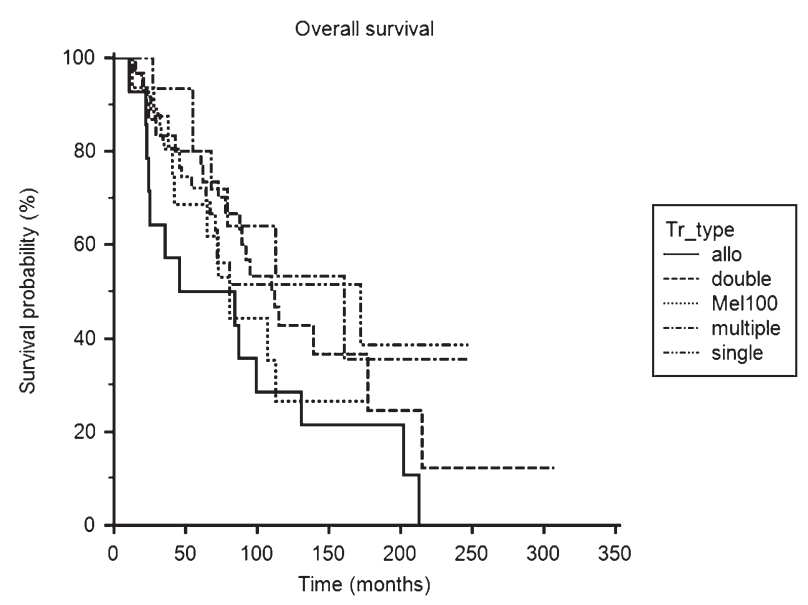

Fig. 2: Overall survival of patients receiving specific type of transplantation (Tr_type: type of transplant used, allo: allogenic transplantation, double: double upfront Mel200, Mel100: upfront Mel100 transplant, multiple: repeated transplantation during the disease course, single: single Mel200 transplant)

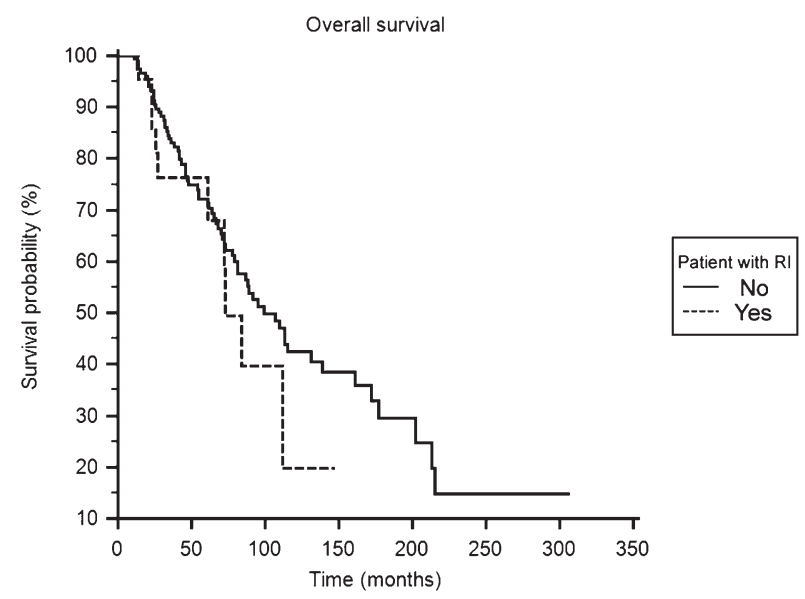

Fig. 4: Overall survival in patients with renal failure versus without renal failure (Yes: patients with stage B myeloma, No: patients with stage A myeloma)

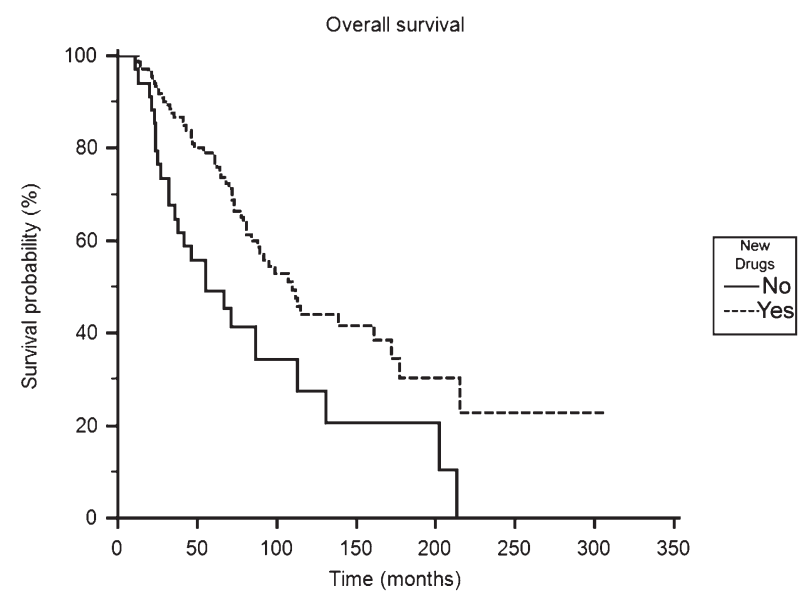

Fig. 6: Overall survival in patients who received new drugs versus those who did not 
the OS was found when patients were divided according to the usage of new drugs - thalidomide, bortezomib and lenalidomide. Patients who had a chance to receive new drugs have anytime during the course of the disease had median survival 110 months versus 55 months in those who did not have any of these drugs $(p=0.0014)$ (figure 6). Results are summarized in table 3.

Tab. 3: Overview of results

\begin{tabular}{|c|c|c|c|c|}
\hline & & $\begin{array}{l}\text { No. Of } \\
\text { patients }\end{array}$ & $\begin{array}{c}\text { Median OS } \\
\text { (months) }\end{array}$ & $\mathrm{p}$ value \\
\hline \multirow[t]{2}{*}{$\begin{array}{l}\text { Patients reaching } \\
\text { CR }\end{array}$} & Yes & 49 & 92 & \multirow{2}{*}{0.31} \\
\hline & No & 134 & 139 & \\
\hline \multirow[t]{2}{*}{$\begin{array}{l}\text { Patients with } \\
\text { renal impairment }\end{array}$} & Yes & 24 & 73 & \multirow{2}{*}{0.22} \\
\hline & No & 159 & 99 & \\
\hline \multirow[t]{2}{*}{$\begin{array}{l}\text { Patients receiving } \\
\text { new drugs }\end{array}$} & Yes & 149 & 110 & \multirow{2}{*}{0.0014} \\
\hline & No & 34 & 55 & \\
\hline \multirow[t]{2}{*}{$\begin{array}{l}\text { Patients with } \\
\text { stage III disease }\end{array}$} & Yes & 87 & 89 & \multirow{2}{*}{0.92} \\
\hline & No & 96 & 112 & \\
\hline
\end{tabular}

\section{Discussion}

Autologous stem cell transplantation has long been a valid treatment option for the treatment of patients with multiple myeloma. However, since 1996, the era has changed facing new challenging treatment options. In concordance with other previously published studies (10-13), the outcome of our patients has been significantly improved when ASCT was used. Here in our analysis we can observe a median survival of 55 months in patients who were transplanted however not treated with novel agents. This roughly represents population treated from 1996 till 2001, when Thalidomide became available at the market in the Czech Republic. Since then we can see a great increase in the survival (110 months at our institution) showing the great benefit of novel agents incorporated into the myeloma treatment. Experience from big clinical trials also support the fact that addition of new drugs improves survival of the patients $(14,15)$. Our data however have a flaw that needs to be taken in consideration. Since more aggressive disease has ever existed but we were unable to describe it by other means than clinical course of the disease in the past, we probably lost many of these patients before they got a chance to profit from the novel agents and vice versa, the good risk patients showing profit from any treatment live long enough to benefit from all ways of treatment. In the view of current data supporting role of novel myeloma stratification according to gene expression profiling showed not only by Arkansas group (16), but also by Mayo group (17), we would be definitely be able to stratify the patients more carefully and avoid unnecessary procedures and treatments. The very same story is probably true when interpreting the data comparing patients with stage II and III disease and patients in CR versus non-CR. We failed to show the benefit of earlier stage of the disease nor the reaching of CR was beneficial for the patients. This is in contradiction with recently published data from long term follow-up of major clinical trials (18). Again, we have to address that at the time of 1996, we did not have ISS staging model nor the CR depth could be evaluated in more sensitive ways as it is possible nowadays. This could explain why the CR rate is not translated into better OS. On the other hand renal failure does not seem to worsen the outcome of the patients. Important issue to address is also allogeneic stem cell transplantation. There were only a few patients undergoing this procedure at our institution and it was almost completely abandoned in 2000, however long-term survivors who are disease free exist even in this small group of 15 patients. This procedure might represent an attractive approach for very high risk patients in the future since no conventional treatment has been successful so far as supported by newly emerged clinical trials $(19,20)$. In the view of current treatment opinions, the role of transplantation in myeloma has been challenged by many authors including our group. However no data so far exist for supporting a withdrawal of autotransplantation from the frontline treatment in patients with myeloma and is still considered to be the important treatment method for the patients under the age of 65 years. Role of allogenic transplantation still remains controversial but new data support this approach.

\section{Acknowledgements}

Supported by research project MZO 00179906 and grants IGA MZ NS 10387-3/2009, 10406-3/2009 and NT $12215-4 / 2011$.

\section{References}

1. Becker N. Epidemiology of multiple myeloma. Recent Results Cancer Res 2011; 183: 25-35.

2. Kyle RA, Rajkumar SV. Criteria for diagnosis, staging, risk stratification and response assessment of multiple myeloma. Leukemia 2009; 23: 3-9.

3. Hoogstraten B, Sheehe PR, Cuttner J, et al. Melphalan in multiple myeloma. Blood. 1967; 30: 74-83.

4. Attal M, Harousseau J-L, Stoppa A-M, et al. A prospective, randomized trial of autologous bone marrow transplantation and chemotherapy in multiple myeloma. N Engl J Med 1996; 335: 91-7.

5. Barlogie B, Attal M, Crowley J, et al. Long-term follow-up of autotransplantation trials for multiple myeloma: update of protocols conducted by the intergroupe francophone du myelome, southwest oncology group, and university of arkansas for medical sciences. J Clin Oncol 2010; 28: 1209-14.

6. Harousseau JL, Attal M, Avet-Loiseau H. The role of complete response in multiple myeloma. Blood 2009; 114: 3139-46.

7. Durie BG, Salmon SE. A clinical staging system for multiple myeloma. Correlation of measured myeloma cell mass with presenting clinical features, response to treatment, and survival. Cancer $1975 ; 36,842-54$

8. Bladé J, Samson D, Reece D, et al. Criteria for evaluating disease response and progression in patients with multiple myeloma treated by high-dose therapy and haemopoietic stem cell transplantation. Myeloma Subcommittee of the EBMT. European Group for Blood and Marrow Transplant. Br J Haematol 1998; 102: $1115-23$. 
9. Slavin S, Nagler A, Naparstek E, et al. Nonmyeloablative stem cell transplantation and cell therapy as an alternative to conventional bone marrow transplantation with lethal cytoreduction for the treatment of malignant and nonmalignant hematologic diseases. Blood 1998; 91: 756-63.

10. Kumar S. Treatment of newly diagnosed multiple myeloma: advances in curren therapy. Med Oncol 2010; 27 Suppl 1: S14-24.

11. Bladé J, Rosiñol L, Sureda A, et al. Programa para el Estudio de la Terapéutica en Hemopatía Maligna (PETHEMA). High-dose therapy intensification compared with continued standard chemotherapy in multiple myeloma patients responding to the initial chemotherapy: long-term results from a prospective randomized trial from the Spanish cooperative group PETHEMA. Blood 2005; 106: 3755-9.

12. Barlogie B, Tricot GJ, van Rhee F, et al. Long-term outcome results of the firs tandem autotransplant trial for multiple myeloma. Br J Haematol 2006; 135 $158-64$.

13. Palumbo A, Bringhen S, Petrucci MT, et al. Intermediate-dose melphalan improves survival of myeloma patients aged 50 to 70 : results of a randomized controlled trial. Blood 2004; 104: 3052-7.

14. Zangari M, van Rhee F, Anaissie E, et al. Eight-year median survival in multiple myeloma after total therapy 2 : roles of thalidomide and consolidation chemotherapy in the context of total therapy 1. Br J Haematol 2008; 141: 433-44.
15. Krejci M, Scudla V, Tothova E, et al. Long-term outcomes of autologous transplantation in multiple myeloma: significant survival benefit of novel drugs in post-transplantation relapse. Clin Lymphoma Myeloma 2009; 9: 436-42.

16. Shaughnessy JD Jr, Zhan F, Burington BE, et al. A validated gene expression model of high-risk multiple myeloma is defined by deregulated expression of genes mapping to chromosome 1. Blood 2007; 109: 2276-84.

17. Kumar SK, Uno H, Jacobus SJ, et al. Impact of gene expression profiling-based risk stratification in patients with myeloma receiving initial therapy with lenalidomide and dexamethasone. Blood 2011; 118: 4359-62.

18. Harousseau JL, Avet-Loiseau H, Attal M, et al. Achievement of at least very good partial response is a simple and robust prognostic factor in patients with multiple myeloma treated with high-dose therapy: long-term analysis of the IFM 99-02 and 99-04 Trials. J Clin Oncol 2009; 27: 5720-6.

19. Bruno B, Rotta M, Patriarca F, et al. Nonmyeloablative allografting for newly diagnosed multiple myeloma: the experience of the Gruppo Italiano Trapianti di Midollo. Blood 2009; 113: 3375-82.

20. Björkstrand B, Iacobelli S, Hegenbart U, et al. Tandem autologous/reduced-intensity conditioning allogeneic stem-cell transplantation versus autologous transplantation in myeloma: long-term follow-up. J Clin Oncol 2011; 29: 3016-22.

Received: $31 / 08 / 2012$

Accepted in revised form: 17/02/2013

\section{Corresponding author:}

Assoc. Prof. Vladimír Maisnar, M.D., Ph.D., University Hospital, Sokolská 581, Hradec Králové, Czech Republic; e-mail: maisnvla@fnhk.cz 